\title{
Spondylometaphyseal dysplasia, Schmidt type
}

INSERM

\section{Source}

INSERM. (1999). Orphanet: an online rare disease and orphan drug data base.

Spondylometaphyseal dysplasia, Schmidt type. ORPHA:93316

Spondylometaphyseal dysplasia, Schmidt type is characterized by short stature, myopia, ,small pelvis, progressive kypho-scoliosis, wrist deformity, severe genu valgum, short long bones, and severe metaphyseal dysplasia with moderate spinal changes and minimal changes in the hands and feet. 